\section{Subglottic Stenosis after Nasal Endotracheal Intubation}

Brit. med. F., 1966, 2, 451-452

Tracheostomy in infants and small children is associated with a high incidence of complications (McClelland, 1965). It has recently been suggested that in this age group prolonged endotracheal intubation with a nasal tube is an acceptable alternative to tracheostomy (McClelland, 1965 ; McDonald and Stocks, 1965 ; Allen and Steven, 1965), and the use of non-irritant polyvinyl chloride tubes is recommended. Jackson Rees has designed a tube specifically for prolonged intubation (Fig. 1) which is now commercially available from Portland Plastics Ltd. in sizes 2.5 to $11 \mathrm{~mm}$.

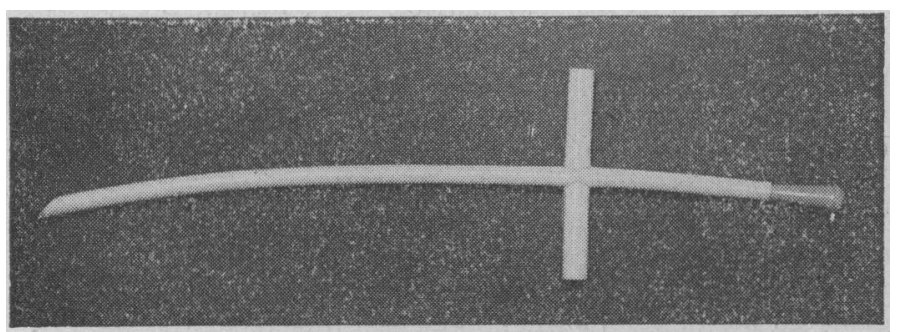

Fig. 1.-Jackson Rees endotracheal tube.

Allen and Steven (1965) suggest that the method may be less suitable in older children, and they are not sure if it is indicated in patients requiring intermittent positive-pressure ventilation, because greater reaction to the tube seems to occur in these cases. They report a series of 61 children treated with prolonged nasal intubation, one of whom developed subglottic stenosis of an apparently permanent character.

We report a similar complication in a 5-year-old boy, weighing approximately $40 \mathrm{lb}$. (18 kg.), who developed severe tetanus, and was treated by prolonged curarization and intermittent positive-pressure ventilation.

\section{Case History}

The patient received a penetrating wound of the right side of his neck while playing with a stick in his garden in East London. The wound seemed to heal cleanly and A.T.S. was not given. He had not been actively immunized against tetanus. Five days later the right side of his neck became stiff, and by the ninth day he was unwell, unsteady on his feet, and had difficulty in swallowing. The next day he was unable to open his mouth, and on the 11th day generalized tetanic spasms occurred and he was admitted to hospital. During the early hours of the 12 th day these spasms became severe enough to cause respiratory embarrassment despite treatment with mephenesin and sedation, and a rubber oral endotracheal tube was passed. Because of the rapidly increasing severity of the disease it was decided that curarization and artificial ventilation were necessary, and he was transferred to this hospital under the care of Dr. Richard Dobbs. He was curarized before transfer and ventilated through a rubber Magill nasal tube, size $5 \mathrm{~mm}$., because the available Jackson Rees tube $(4.5 \mathrm{~mm}$.) was found to be too short for safety during transit. After his transfer the rubber tube was removed and a $5.5-\mathrm{mm}$. Jackson Rees tube substituted (after confirming that a 5-mm. Jackson Rees tube was too short). Ventilation was maintained by a Barnet ventilator with adequate humidification, and an intensive nursing regimen involving aseptic tracheal toilet was instituted (Taylor, Simpson, and Aspinall, 1965).

Curarization and intermittent positive-pressure ventilation were maintained until the 20th day after injury; during this period the tube became dislodged, necessitating a further intubation (making a total of at least six intubations). As the effect of the tubocurarine wore off it became obvious that severe spasms were still occurring and that the regimen must be continued, and it was decided that an elective tracheostomy should be performed. On the 21 st day, after nine days' naso-endotracheal intubation, tracheostomy was performed under general anaesthesia and a 27 F.G. cuffed Bassett plastic tracheostomy tube inserted. Laryngoscopy (T. H. T.) showed the larynx distorted by oedema, with a central circular opening corresponding in size to the endotracheal tube just withdrawn. Laryngoscopy was repeated the next day by the same person, to whom the larynx and upper part of the trachea now appeared normal.

Apart from one further trial without curare from the 26th to 29 th days, the regimen was continued until the 33rd day, when curare was finally stopped. The patient was weaned completely from the ventilator by the 36 th day and showed no further manifestations of tetanus. The cuffed tracheostomy tube was left in place for a further seven days, during which time the naso-gastric tube was removed and normal swallowing was established. On the 43rd day decannulation was carried out in order to determine whether or not the upper airway was patent; it was found to be completely occluded, so a 24 F.G. Bassett tracheostomy tube was inserted. The cuff of this smaller tube was not inflated, and phonation, which occurred on the 46th day, demonstrated that an airway had been re-established. The next day it was decided to visualize his larynx and upper trachea under general anaesthesia, and to substitute a fenestrated silver cannula for the plastic tracheostomy tube. Laryngoscopy revealed a normal larynx with an apparently complete subglottic obstruction. After dilatation with an infant Negus bronchoscope (6 mm. maximum outside diameter) a normal-sized airway was established, but with a raw area immediately below the left vocal cord. During the next eight days the tracheostomy tube was plugged intermittently for increasing periods and his voice improved. $X$-ray examination of the soft tissue of the neck at this time (Figs. 2 and 3 ) showed subglottic narrowing and a radio-opaque foreign body at the level of the narrowing but to the right of the trachea and deep to the site of entry of the original wound. This foreign body presumably was carried in at the time of the original injury and has yet to be removed. Bronchoscopy on the 55th day demonstrated a circumferential stenosis of the upper trachea with a lumen of 2 to $3 \mathrm{~mm}$. diameter. This was dilated to approximately $5 \mathrm{~mm}$., and a course of antibiotic and steroid therapy was started.

The subsequent management of this case was undertaken by the department of otorhinolaryngology, and was based upon intermittent bronchoscopic dilatations and the use of fenestrated or Negus silver tracheostomy cannulae with speaking-valves to encourage phonation. In the 12th week of the boy's illness improvement of his airway

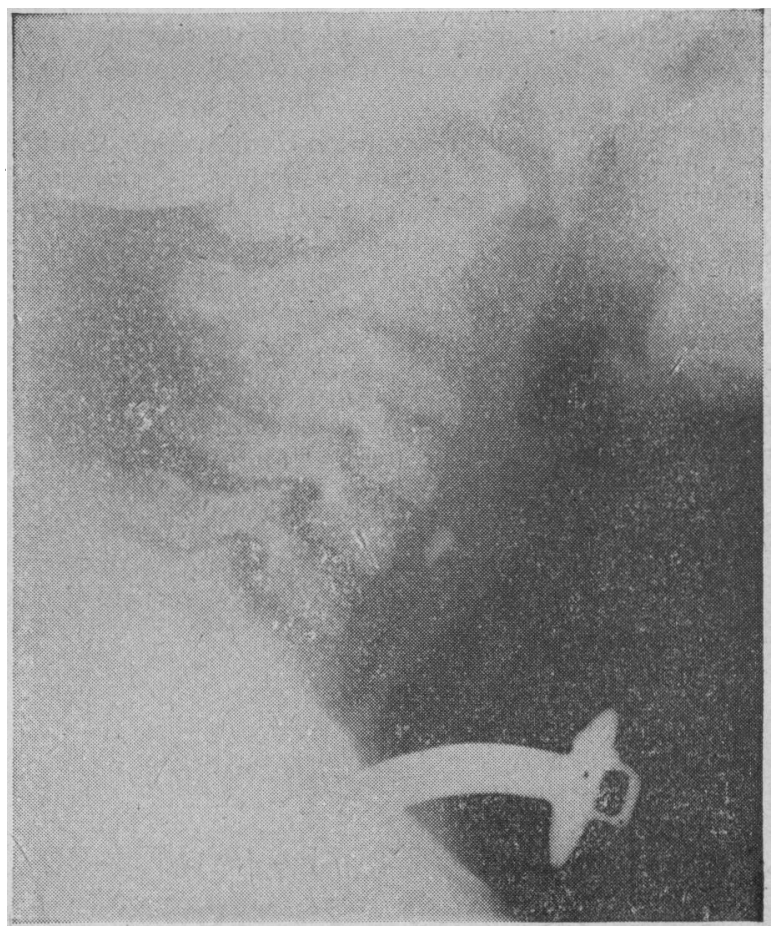

FIG. 2.-Lateral radiograph of the patient's neck to show narrowing of the trachea in the subglottic region, and also the radio-opaque foreign body. 
was so marked that decannulation was attempted again, but with the closure of the tracheostomy stoma it became obvious that his airway was inadequate, and he was recannulated under general anaesthesia some 20 hours later. One week after this the subglottic stenosis was at its worst, with a lumen of about $1 \mathrm{~mm}$. However, there has since been progressive improvement and he now has a normal speaking-voice with a Negus tracheostomy cannula. It is hoped that this can be removed in the near future.

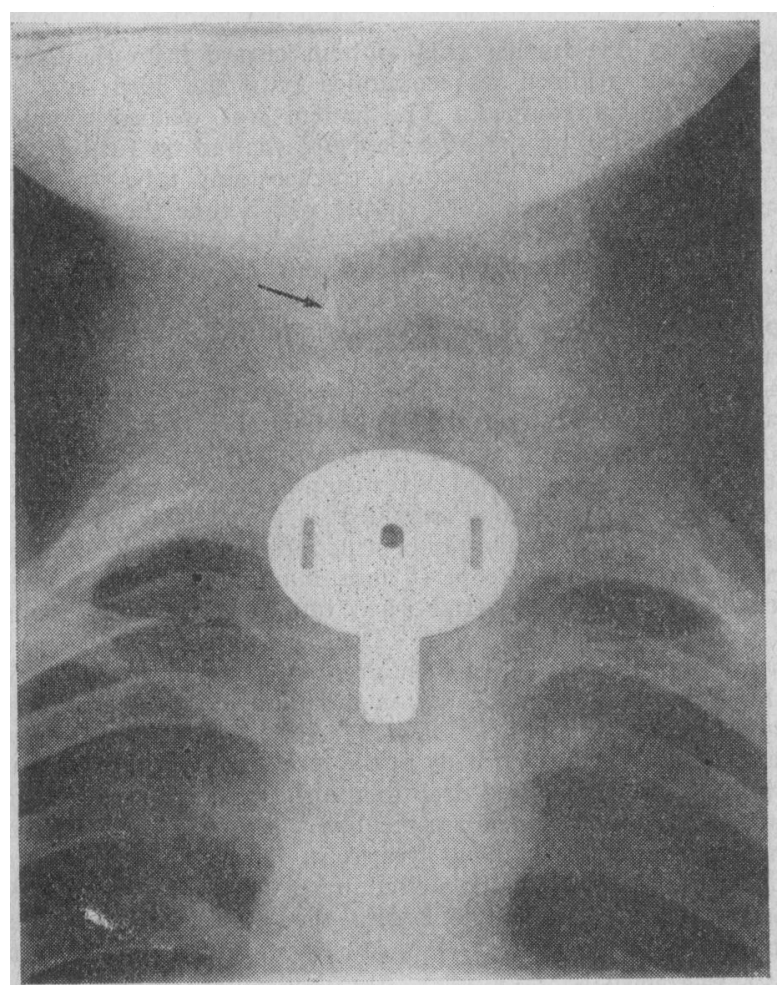

FIG. 3.-Antero-posterior radiograph of the patient's neck to show the position of the radio-opaque foreign body.

\section{COMMrNT}

The patient has survived a severe and potentially fatal attack of tetanus, but as a direct result of our management he has been left with a major complication which could endanger his life.

Tracheal stenosis is a not uncommon complication of tracheostomy in young children. However, in this case the situation of the stenosis, just below the vocal cords and significantly above the level of the tracheostomy stoma (Fig. 2), must implicate the endotracheal tube as a major factor in the production of the lesion.

The normal appearance of the larynx and upper trachea one day after extubation indicates that there was no clear evidence of trauma related either to the multiple intubations to which this boy was subjected in the early days of treatment or to the continued presence of the endotracheal tube, so we can only speculate regarding the cause of the stenosis. We feel that diversion of the air-stream from the upper part of the trachea immediately after extubation contributed to the develop- ment of this stenosis by allowing potentially infected secretions to collect above the cuffed tracheostomy tube. Despite nursing the patient in a head-down position with continuous oropharyngeal suction, some secretions must have collected in the subglottic region and there pooled on mucosa, the normal functions of which must have been affected by prolonged contact with even a "non-irritant" endotracheal tube. In this context it is interesting to note the work of Little and Parkhouse (1962), who suggested, after animal experiments, that some polyvinyl chloride products can be irritant to living tissue.

In retrospect, it could be that our policy of carrying out a tracheostomy after nine days' endotracheal intubation was wrong, and that this case might have been better managed by either method alone.

Other factors must be considered in the production of this stenosis. (1) The trachea could have been damaged by the original penetrating injury, the track of which must have passed close to the site of the stenosis because of the situation of the entry wound and the position of the retained foreign body (Figs. 2 and 3). (2) The trachea could have been damaged by movement transmitted to the nasotracheal tube by intermittent positive-pressure ventilation. It is worth noting here that the complication rate rises when intermittent positive-pressure ventilation replaces spontaneous ventilation, whether applied via a tracheostomy or a nasotracheal tube (McClelland, 1965; Allen and Steven, 1965). (3) The trachea could have been damaged by the use of an endotracheal tube of too large a size, as McDonald and Stocks (1965) and Allen and Steven (1965) suggested in their cases. There is no evidence that in our case any of the tubes used was of excessive diameter.

ADDENDUM.- Since this paper was submitted for publication the tracheostomy tube was finally removed in the 19th week, having been in place for 16 weeks. Laryngoscopy at that time showed some residual stenosis, but with a 4-mm. opening, which was thought to be adequate if the child remained in hospital. Laryngoscopy four weeks later showed an almost normal larynx and upper trachea with a small residual band of tissue anteriorly. The patient recovered a normal speaking-voice and has gone home.

We wish to thank Dr. R. Dobbs for permission to publish the details of this case, which was admitted under his care. Mr. Andrew Morrison has undertaken the management of the tracheal stenosis. Mr. Ruddick, of the London Hospital Photographic Department, took the photographs.

T. H. TAYLOR, M.B., B.S., F.F.A. R.C.S.

D. A. Nightingale, B.A., M.B., B.CHIR., F.F.A. R.C.S., D.A.

B. R. SIMPSON, D.PHIL., M.R.C.S., L.R.C.P., D.OBST.R.C.O.G., D.A., F.F.A. R.C.S.

Department of Anaesthetics,

The London Hospital.

\section{REFERENCES}

Allen, T. H., and Steven, I. M. (1965). Brit. 7. Anaesth., 37, 566. Little, K., and Parkhouse, J. (1962). Lancet, 2, 857.

McClelland, R. M. A. (1965). Brit. med. f., 2, 567

McDonald, I. H., and Stocks, J. G. (1965). Brit. ₹. Anaesth., 37, 161. Taylor, T. H., Simpson, B. R., and Aspinall, K. W. (1965). Lancet, 1, 89. 